\title{
In vivo optical-resolution photoacoustic computed tomography with compressed sensing
}

\author{
Jing Meng, ${ }^{1,3}$ Lihong V. Wang, ${ }^{2}$ Dong Liang, ${ }^{1}$ and Liang Song,* \\ ${ }^{1}$ Institute of Biomedical and Health Engineering, Shenzhen Institutes of Advanced Technology, Chinese Academy of Sciences, \\ 1068 Xueyuan Boulevard, Nanshan, Shenzhen 518055, China \\ ${ }^{2}$ Department of Biomedical Engineering, Washington University in St. Louis, St. Louis, Missouri 63130, USA \\ ${ }^{3}$ College of Computer Science, Qufu Normal University, 80 Yantai Road North, Donggang, Rizhao 276826, China \\ ${ }^{*}$ Corresponding author: liang.song@siat.ac.cn
}

Received September 11, 2012; accepted October 1, 2012;

posted October 4, 2012 (Doc. ID 176019); published November 6, 2012

\begin{abstract}
Optical-resolution photoacoustic microscopy is becoming a powerful research tool for studying microcirculation in vivo. Moreover, ultrasonic-array-based optical-resolution photoacoustic computed tomography (OR-PACT), providing comparable resolution at an improved speed, has opened up new opportunities for studying microvascular dynamics. In this Letter, we have developed a compressed sensing with partially known support (CS-PKS) photoacoustic reconstruction strategy for OR-PACT. Compared with conventional backprojection reconstruction, the CS-PKS strategy was shown to produce high-quality in vivo OR-PACT images with threefold less measurement data, which can be leveraged to improve the data acquisition speed and costs of OR-PACT systems. (C) 2012 Optical Society of America

OCIS codes: $170.5120,170.3880,170.0180$.
\end{abstract}

Photoacoustic tomography (PAT) is capable of acquiring images of various biological structures from organelles to organs in vivo [1]. In particular, photoacoustic microscopy (PAM), one form of PAT, has become a vital tool for imaging microvasculature, oxygen saturation, etc., in various biomedical applications. To achieve micrometer or even submicrometer lateral resolution, opticalresolution PAM (OR-PAM) has been developed by mechanically scanning a focused laser beam together with a confocal ultrasonic transducer [2]. However, the data acquisition (DAQ) of OR-PAM is time consuming due to the required large number of scanning steps. Recently, to improve the imaging speed of OR-PAM, we developed multifocal OR photoacoustic computed tomography (ORPACT) with ultrasonic array detection [3]. With an eightchannel DAQ card, OR-PACT was approximately three to four times faster in image acquisition than OR-PAM. However, unlike conventional array-based photoacoustic computed tomography (PACT) that acquires a full B-scan with one laser shot, OR-PACT requires 25 laser shots for one B-scan, leading to a greatly increased measurement data volume [3]. This, together with its $6: 1$-multiplexingDAQ scheme, has prevented OR-PACT from realizing its full potential for high-speed imaging-20-fold faster than OR-PAM. In addition, densely packed high-frequency ultrasonic arrays (with a pitch $\sim 1 / 2$ of the ultrasonic center wavelength), needed for obtaining high-quality ORPACT images with backprojection (BP) reconstruction, are still difficult to fabricate and can be quite expensive. While the use of a sparse ultrasonic array for PACT has also been explored, the image quality was somewhat compromised with conventional BP reconstruction [4].

Compressed sensing (CS) can recover compressible signals from undersampled measurements. It has been successfully applied to imaging modalities such as magnetic resonance imaging [5], computed tomography [6], and diffuse optical tomography [7]. Recently, with numerical simulation and tissue phantoms, Provost and Lesage demonstrated the feasibility to use CS for PACT
[]. Guo et al. then developed CS-based PACT in vivo in the time domain [9]. In our previous studies, CS-based PACT in vivo in both time and frequency domains were implemented, and a novel reconstruction strategy-CS with partially known support (CS-PKS) - was developed for PACT [10]. In this Letter, we have further developed our CS-PKS reconstruction strategy for OR-PACT, which is of significant difference from conventional PACT in system architecture. We have found that, using CS-PKS, high-quality images can be reconstructed for in vivo ORPACT with threefold less measurement data than that required by BP, suggesting CS-PKS is a promising way to help OR-PACT realize its full potential for high-speed, high-resolution imaging.

Figure 1 illustrates the basic principles of OR-PACT. To excite photoacoustic signals, a tunable dye laser (Sirah, Germany) pumped by a $Q$-switched Nd:YLF laser (EdgeWave, Germany) was used. A linear microlens array (SUSS MicroOptics, Switzerland) was employed to provide 20 focused optical illumination beams spaced at $250 \mu \mathrm{m}$ (Fig. 1, inset A) (each beam of a fluence $\sim 8 \mathrm{~mJ} / \mathrm{cm}^{2}$ per pulse on the skin surface, well below the $20 \mathrm{~mJ} / \mathrm{cm}^{2}$ ANSI laser safety limit). A linear ultrasonic array (center frequency, $30 \mathrm{MHz}$; receive-only bandwidth, $70 \%$ ), with 48 transducer elements $(82 \mu \mathrm{m} \times 2 \mathrm{~mm})$ spaced at $100 \mu \mathrm{m}$, was used to simultaneously detect

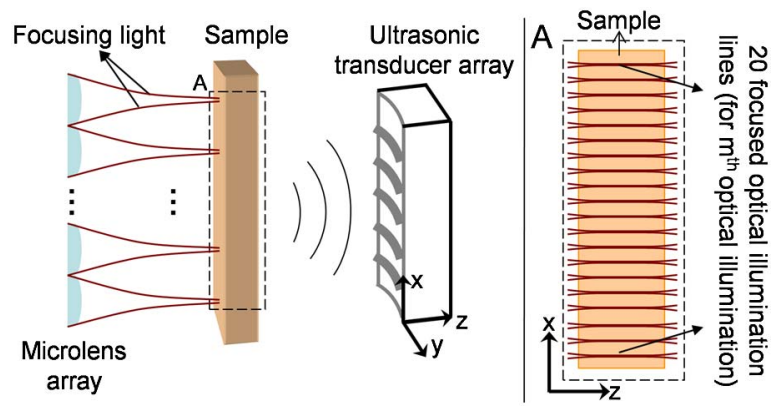

Fig. 1. (Color online) Schematic illustration of OR-PACT. 
photoacoustic emissions from all illumination beams. After each laser shot, the sample (or animal) was scanned along $x$ at a step size of $10 \mu \mathrm{m}$, close to the spot size of one focused laser beam. As a result, with 25 laser shots, the image data of a $5 \mathrm{~mm} \times 2.5 \mathrm{~mm}(x-z)$ cross section were acquired. For three-dimensional (3D) imaging, the sample was scanned along $y$ at a step size of $10 \mu \mathrm{m}$ to repeat the above acquisition for a series of $x-z$ cross sections. Thus, for each $x-z$ cross-section image, a data set of $48 \times 200 \times 25$ was acquired, $\sim 2.5$-fold larger than the $500 \times 200$ OR-PAM B-scan data set. Further details on the OR-PACT system can be found in [3].

The flowchart of our reconstruction strategy for OR-PACT is illustrated in Fig. 2. One 3D OR-PACT image consists of a series of B-scans. To obtain each B-scan, three major steps are involved. (1) Upon one laser shot (optical illumination), reconstruct the $x-z$ cross-section image $(500 \times 200)$ with CS-PKS or BP, using detected photoacoustic signals from the ultrasonic array. (2) Reconstruct the pre-B-scan signals along the 20 optical illumination lines in the cross section. Specifically, for each illumination line, the data within $\Delta x(150 \mu \mathrm{m}$ along $x$, which is slightly greater than the lateral resolution of the ultrasonic array) of this line are integrated and considered as the true signals along this illumination line. (3) Put the reconstructed pre-B-scan $(20 \times 200)$ to the corresponding columns of the preset B-scan matrix. After 25 times of optical illumination (25 laser shots), one full B-scan image $(500 \times 200)$ can be reconstructed. An example showing the B-scan reconstruction process above is depicted in Fig. 2 .

In the reconstruction of the $x-z$ cross-section image above, i.e., step 1, either BP or CS-PKS can be used. With BP, the detected photoacoustic signals are directly

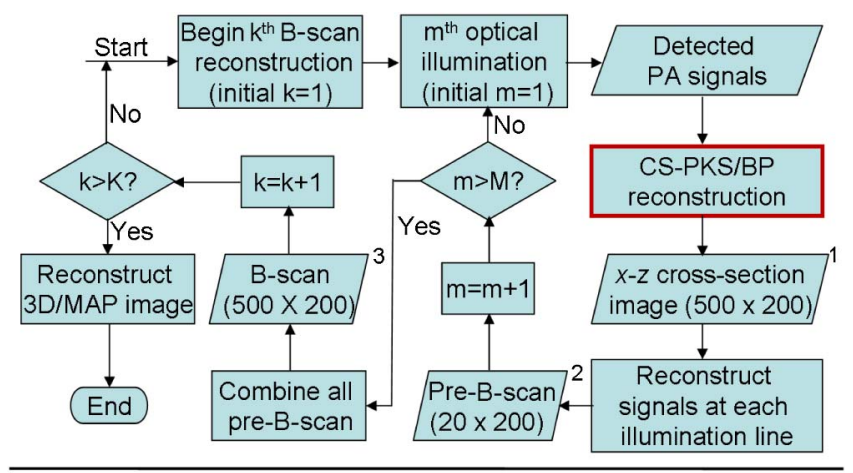

Example: Reconstruction of a representative B-scan

1. $x-z$ cross-section image
for $m^{\text {th }}$ optical illumination

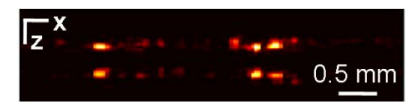

2. Pre-B-scan image for $\mathrm{m}^{\text {th }}$ optical illumination

3. B-scan $\mathrm{k}$ (consisting of M pre-B-scans)

ig. 2. (Color online) Flowchart of our reconstruction strategy for OR-PACT. K, number of B-scans; M, the number of times of optical illumination (25 in this Letter) for each B-scan; PA, photoacoustic; MAP, maximum amplitude projection. backprojected to recover the initial photoacoustic pressures. With CS, the sparsity of PACT images (in certain domains) is utilized to enable reconstruction with spatially undersampled measurements. With CS-PKS, part of the nonzero signals' locations in the sparse transform domain are utilized, as partially known support (PKS), to further improve the CS reconstruction. Mathematically, the CS-PKS-based image reconstruction can be expressed as the following optimization problem:

$$
\arg \min _{\mathbf{x}} F=\|\Phi \mathbf{x}-\mathbf{y}\|_{2}^{2}+\alpha\left\|\mathbf{x}_{\Delta}\right\|_{1}+\beta \mathbf{T V}\left(\Psi^{-1} \mathbf{x}\right),
$$

where $\mathbf{x}$ is the representation of the image to be reconstructed in the sparse transform domain, $\mathbf{y}$ represents the detected photoacoustic signals, $\Phi$ represents the CS matrix, $\Psi$ represents the sparse transform (a four-level Daubechies wavelet transform in this Letter), and $\alpha$ and $\beta(0.15$ and 0.25 , respectively, in this Letter) are the regularization parameters determining the trade-off between the data consistency and the sparsity. In Eq. (1), the objective function $F$ consists of three parts. The first part represents the square error between the estimated measurements from the reconstructed image and the experimentally acquired measurements, the second part represents the $l_{1}$ norm of $\mathbf{x}$ with PKS, and the third part represents the total variation penalty of the image. To solve Eq. (1), an iterative reweighed conjugate gradient descent method was used, as discussed in detail in [10].

To validate our CS-PKS reconstruction strategy for $\overline{\mathrm{OR}}$ PACT, the ear of an anesthetized nude mouse weighing $\sim 20 \mathrm{~g}$ was imaged in vivo. The imaging procedure was the same as that described in our previous publication [3]. All experimental animal procedures were carried out in compliance with protocols approved by the Animal Studies Committee of Washington University.

Figure 3 shows the reconstructed OR-PACT images of the mouse ear microvasculature. Figures $3(\mathrm{~A})$ and $\underline{3(\mathrm{~B})}$ are the maximum amplitude projection (MAP) imagesthe maximum photoacoustic amplitudes projected along the depth direction to the surface of the earreconstructed by $\mathrm{BP}$ with data from all 48 transducer elements and from only 16 uniformly distributed elements. Figure 3(C) is the MAP image reconstructed by CS-PKS with data from the same 16 transducer elements. Figures $3(\mathrm{a})-3(\mathrm{c})$ are enlarged images of the rectangular boxes in Figs. 3(A)-3(C). From these reconstructed images, it can be clearly seen that, (1) using conventional $\mathrm{BP}$, the image reconstructed with data from only 16 transducer elements is significantly worse than that reconstructed with data from all 48 transducer elements. In the former case, the contrast-to-noise ratio (CNR) is severely deteriorated, and details of the image are lost [as indicated by the arrows in Figs. 3(a) and 3(b)]. (2) Using CS-PKS, however, the image reconstructed with data from only 16 transducer elements exhibits a CNR and detailed image features [as indicated by the arrows in Figs. 3(a) and 3(c)] comparable to that reconstructed by BP with data from all 48 transducer elements, i.e., the "gold standard" in this Letter.

To quantitatively evaluate the reconstructed images, we computed the histograms of the difference imagesimages obtained by subtracting the control from the 

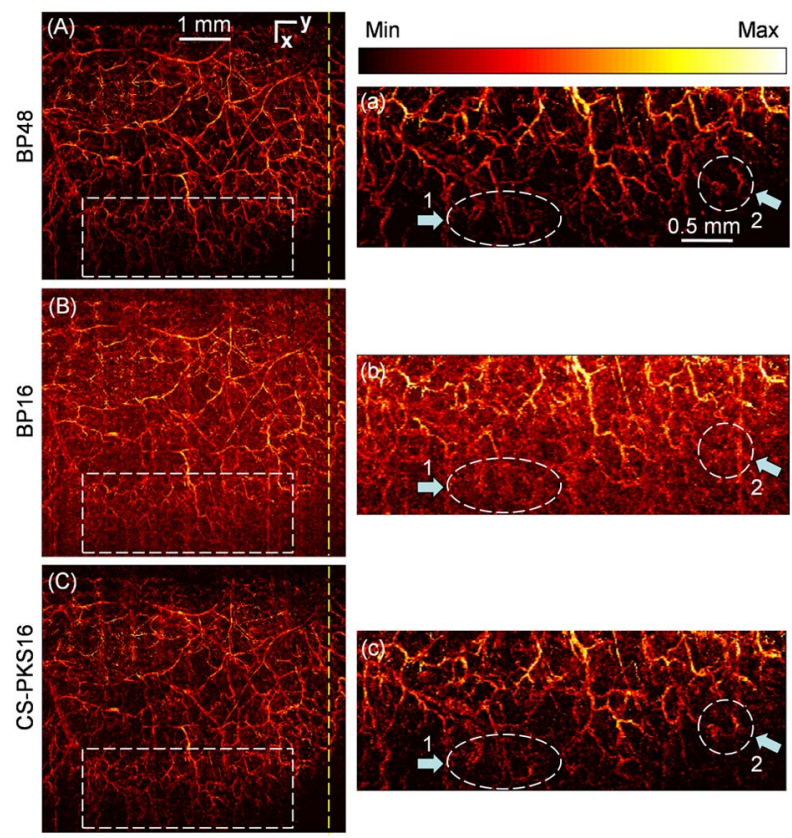

Fig. 3. (Color online) In vivo OR-PACT images of a mouse ear microvasculature. (A), (B) MAP images reconstructed by BP with data from 48 and 16 transducer elements, respectively. (C) MAP image reconstructed by CS-PKS with data from 16 transducer elements. (a), (b), (c) Selected regions from (A), (B), (C), respectively, as indicated by the rectangular boxes. The number 48 or 16 after each reconstruction method indicates that the reconstruction is performed with data from either 48 or 16 transducer elements. The color scale represents relative optical absorption.

reconstructed images and then taking the absolute value [Figs. 4(A) and 4(B)] (all images were first normalized by dividing by their corresponding maximum value). The control used in our calculation was the image reconstructed by BP with data from all 48 transducer elements. Figures $\underline{4(\mathrm{~A})}$ and $\underline{4(\mathrm{~B})}$ are the histograms of the difference images between the control and reconstructed images by BP and CS-PKS, respectively, with data from 16 transducer elements. It can be seen that the number of counts lying in the lower amplitude ranges in Fig. 4(B) is larger than that in Fig. 4(A), indicating that the image reconstructed by CS-PKS possesses higher fidelity. Further, localized comparisons were performed by plotting the photoacoustic amplitudes along chosen lines in the MAP images of Figs. 3(A)-3(C) [Fig. 4(C)]. The CNRs of selected signal peaks were also computed (Fig. 4, inset) (4.0-5.0 mm along $x$ was used as an estimation of the background). It can be seen that, overall, the image reconstructed by CS-PKS has a significantly higher CNR than that obtained with BP.

In summary, for the first time to our knowledge, we have developed and adapted a CS-PKS reconstruction strategy for OR-PACT. Using this strategy, even with heavily spatially undersampled measurements (using data from only 16 transducer elements compared with that from 48 elements in previous OR-PACT), high-quality OR-PACT images can still be reconstructed. As a result, without any modifications of the system hardware, the DAQ speed of OR-PACT can be improved threefold by reducing the DAQ multiplexing from $6: 1$ to $2: 1$. With this
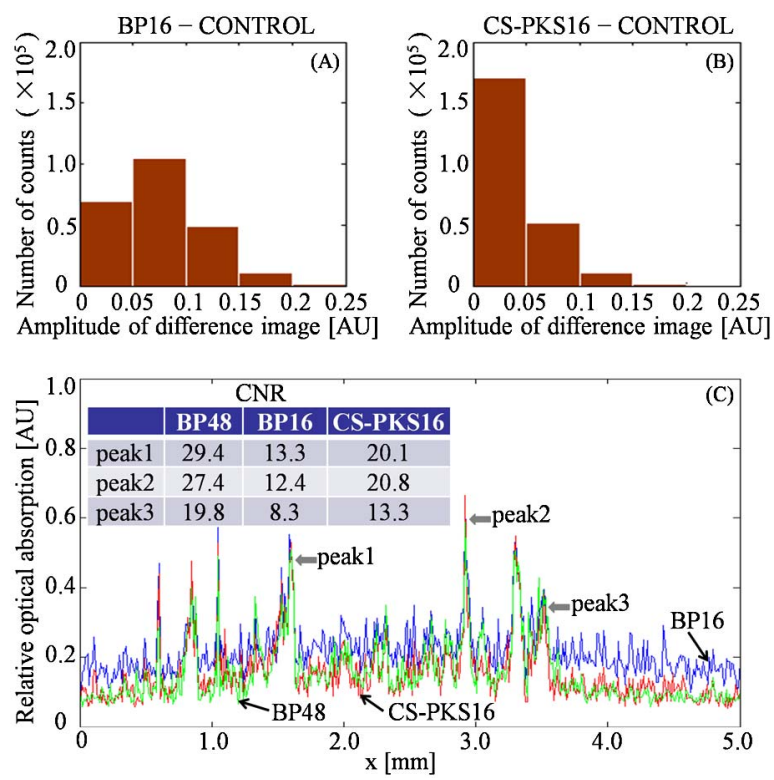

Fig. 4. (Color online) (A), (B) Histograms of the amplitudes of the difference images using the image reconstructed by $\mathrm{BP}$ with data from all 48 transducer elements as the control. (C) Photoacoustic amplitudes along the chosen yellow lines in the MAP images of Figs. 3(A)-3(C). (Inset) CNRs of selected signal peaks. The number 48 or 16 after each reconstruction method indicates that the reconstruction is performed with data from either 48 or 16 transducer elements.

strategy, our OR-PACT can achieve an imaging speed $\sim 10$ times faster than conventional OR-PAM. In the future, a sparse ultrasonic array may be used to reduce the cost of OR-PACT systems as well. The results in this Letter suggest that the use of CS-based reconstruction strategies-in particular, CS-PKS-in combination with high-frequency ultrasonic arrays can be a promising way to develop high-speed, low-cost OR-PACT for imaging various biomedical dynamics in vivo.

This work was supported in part by the National Natural Science Foundation of China grant no 61205203, the Guangdong Innovation Research Team Fund for Low-Cost Healthcare Technologies (GIRTFLCHT), and the Shenzhen Development and Reform Commission grant no. [2012] 1065.

\section{References}

1. L. V. Wang and S. Hu, Science 335, 1458 (2012).

2. K. Maslov, H. F. Zhang, S. Hu, and L. V. Wang, Opt. Lett. 33, 929 (2008).

3. L. Song, K. Maslov, and L. V. Wang, Opt. Lett. 36, 1236 (2011).

4. P. Ephrat, L. Keenliside, A. Seabrook, F. S. Prato, and J. J. L. Carson, J. Biomed. Opt. 13, 054052 (2008).

5. M. Lustig, D. Donoho, and J. M. Pauly, Magn. Reson. Med. 58, 1182 (2007).

6. G. H. Chen, J. Tang, and S. Leng, Med. Phys. 35, 660 (2008).

7. D. Han, J. Tian, K. Liu, J. Feng, B. Zhang, X. Ma, and C. Qin, IEEE Trans. Biomed. Eng. 57, 2564 (2010).

8. J. Provost and F. Lesage, IEEE Trans. Med. Imaging 28, 585 (2009).

9. Z. Guo, C. Li, L. Song, and L. V. Wang, J. Biomed. Opt. 15, 021311 (2010).

10. J. Meng, L. V. Wang, L. Ying, D. Liang, and L. Song, Opt. Express 20, 16510 (2012). 